\title{
Challenges for the Adaptive Gain Integrating Pixel Detector (AGIPD) design due to the high intensity photon radiation environment at the European XFEL
}

\author{
J. Becker*, L. Bianco, P. Göttlicher, H. Graafsma, H. Hirsemann, S. Jack, A. Klyuev, \\ S. Lange A. Marras, U. Trunk \\ DESY, Hamburg, Germany \\ E-mail: Julian.Becker@desy.de
}

R. Klanner, J. Schwandt, J. Zhang

University of Hamburg, Germany

R. Dinapoli, D. Greiffenberg, B. Henrich, A. Mozzanica, B. Schmitt, X. Shi

PSI, Villigen, Switzerland

M. Gronewald, H. Krüger

University of Bonn, Germany

\begin{abstract}
The European X-ray Free Electron Laser (XFEL) is a new research facility currently under construction in Hamburg, Germany. With a pulse length of less than $100 \mathrm{fs}$ and an extremely high luminosity of 27000 flashes per second the European XFEL will have a unique time structure that demands the development of new detectors tailored to the requirements imposed by the experiments while complying with the machine specific operation parameters. The Adaptive Gain Integrating Pixel Detector (AGIPD) is one response to the need for large 2D detectors, able to cope with the $4.5 \mathrm{MHz}$ frame rate, as well as with the high dynamic range needed by XFEL experiments ranging from single photons to more than $10^{4} 12 \mathrm{keV}$ photons per pixel per pulse. In addition it has to withstand doses of up to 1 GGy over three years.
\end{abstract}

The 21st International Workshop on Vertex Detectors

16-21 September 2012

Jeju, Korea

\footnotetext{
* Speaker.
} 


\section{Introduction} as possible.
The European X-ray Free Electron Laser (XFEL) 42 $[1,2]$ will provide ultra short, highly coherent X-ray 43 pulses which will revolutionize scientific experiments in 44 a variety of disciplines spanning physics, chemistry, ma- 45 terials science, and biology.

Dedicated fast 2D detectors for the European XFEL are being developed, one of which is the Adaptive Gain 47 Integrating Pixel Detector (AGIPD) [3, 4, 5], developed 48 by a collaboration between DESY, the University of Hamburg, the University of Bonn (all in Germany) and the 49 Paul Scherrer Institute (PSI) in Switzerland.

\section{Requirements}

One of the differences between the European XFEL 55 and other free electron laser sources is the high pulse ${ }_{56}$ repetition frequency of $4.5 \mathrm{MHz}$. The European XFEL ${ }_{57}$ will provide pulse trains, consisting of up to $2700 \mathrm{x}$-ray 58 pulses of less than $100 \mathrm{fs}$ duration, separated by $220 \mathrm{~ns}$ (600 $\mu$ s in total), followed by an idle time of $99.4 \mathrm{~ms}, 60$ resulting in a supercycle of $10 \mathrm{~Hz}$ and 27000 pulses per ${ }_{6}$ second. The energy of the $\mathrm{x}$-rays will be tunable in a 62 range depending on the experimental station. Together ${ }_{63}$ the beamlines will cover the energy range from a few 64 hundreds of $\mathrm{eV}$ to several tens of $\mathrm{keV}$.

As XFELs develop an enormous power density at 66 the sample location, only very few samples will survive 67 illumination. Most samples will be destroyed by the $\mathrm{x}-68$ ray pulse [6], making the acquisition of the diffracted 69 patterns from a single pulse mandatory. Obviously it is 70 desirable to acquire as many of the 2700 images per train 71

As the dynamic range required by common exper- 73 iments is very large, from many thousands of photons 74 close to the central beam to essentially single photon 75 events at large angles, the dynamic range of the em- 76 ployed detection system has to be large as well.

It should be noted that not only the high number ${ }^{77}$ of photons close to the central beam and in Bragg spots 78 needs to be measured correctly, but the reliable detection 79 of single photons and their discrimination against background is equally, if not even more, important.

Additionally, all detector systems for the European XFEL need to be sufficiently radiation hard to survive an estimated dose of $10^{16} 12.4 \mathrm{keV}$ photons, corresponding to 1 GGy at the entry window [7], during 3 years of operation.

\section{The Adaptive Gain Integrating Pixel Detector (AGIPD)}

AGIPD is based on hybrid pixel technology and aims at imaging in the energy range between 3 and $15 \mathrm{keV}$. The current design goals of the newly developed Application Specific Integrated Circuit (ASIC) with independent dynamic gain switching amplifiers in each pixel are (for each pixel) a dynamic range of more than $10^{4}$ $12.4 \mathrm{keV}$ photons in the lowest gain, single photon sen sitivity in the highest gain, and operation at $4.5 \mathrm{MHz}$ frame rate. An external veto signal can be provided to maximize the number of useful images by overwriting any image previously recorded during the pulse train.

Due to the special pulse structure of the European XFEL, it is necessary to store the acquired images inside the pixel circuit area during the pulse train. A compromise had to be found between storing many images, requiring a large pixel area, and high spatial resolution, requiring small pixel sizes $[3,4]$. The image data is read out and digitized in the $99.4 \mathrm{~ms}$ between pulse trains.

The AGIPD will feature a pixel size of $(200 \mu \mathrm{m})^{2}$ which is sufficient to accommodate an analog memory for 352 images. For many experiments using particle injection mechanisms the memory of AGIPD should be sufficient, as particle hit rates are currently below $10 \%$ (see [8] and references therein). The impact of the limited number of storage cells on X-ray Photon Correlation Spectroscopy (XPCS) as intended to be used on the MID station [9] depends on the properties of the sample and has been investigated in [10].

\subsection{Sensor}

The design of the AGIPD sensor is especially challenging, as it needs to be very radiation hard (approxi- 


\begin{tabular}{c|c|c} 
Property & Specification & Comment \\
\hline readout electrodes & $\mathrm{p}^{+}$implants & hole collection \\
bulk doping & $3-8 \mathrm{k} \Omega \cdot \mathrm{cm}$ & n-type bulk material \\
dimension & $107.6 \mathrm{~mm} \times 28 \mathrm{~mm}$ & large monolithic sensors reduce the overall dead area \\
thickness & $500 \mu \mathrm{m}$ & $>90 \%$ quantum efficiency at $12.4 \mathrm{keV}$ \\
effective entry window thickness & $<2.5 \mu \mathrm{m}$ & important for QE at lower energies \\
bias voltage & $>500 \mathrm{~V}$ & minimizes impact of plasma effects
\end{tabular}

Table 1: Important major specifications of the AGIPD sensor.

age suppresses the impact on the scientific imaging. It is expected that plasma effects will manifest themselves in the parts of diffraction patterns close to the central beam and the HORUS simulation tool $[19,20,21]$ was developed to evaluate the scientific impact.

\subsection{Application Specific Integrated Circuit (ASIC)}

The AGIPD readout ASICs will be manufactured in IBM $130 \mathrm{~nm}$ CMOS technology. Several small (16x16 pixels) prototypes have been produced using Multi Project/ Wafer (MPW) runs, both to test the technology characteristics and to evaluate the best architectural solutions to be employed [22]. Radiation-hard design techniques are employed, including the use of Enclosed Layout Transistors (ELTs) and guard rings around critical devices.

Design issues for the full scale (64x64 pixels) chip are settled, and a submission to the foundry is scheduled beginning of 2013 .

A block diagram of the full scale $(64 \times 64$ pixels $)$ chip AGIPD 1.0 is shown in figure 1. The typical signal path comprises charge generation and transport within the sensor, charge collection and (amplified) charge to voltage conversion in the adaptive gain amplifier, Correlated Double Sampling (CDS) of the amplifier output voltage by the CDS stage, signal storage in the analog memory cells, readout of the storage cells and transfer of the signal to the outside world via differential lines for the analog signals. Commands and clock signals are received via LVDS lines.

\subsubsection{Noise performance}

For pristine chips under standard operation condi- 


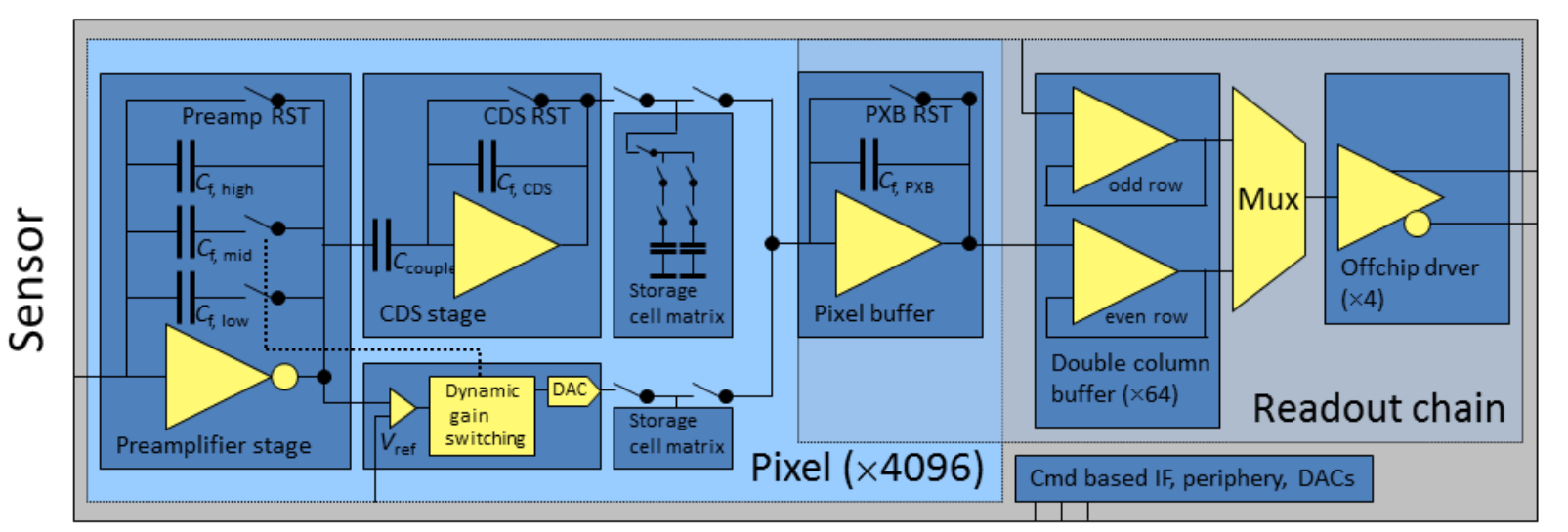

\section{External clock and command signals}

Figure 1: Block diagram of the circuitry on the AGIPD 1.0 chip. Reset switches are abbreviated RST and the Correlated Double Sampling stage CDS. The double column buffer works in an interleaved way by connecting only to odd or even rows in each column, thereby relaxing the timing constraints on the buffer.

\footnotetext{
${ }^{3}$ Standard operating conditions employ about $100 \mathrm{~ns}$ integration 179 time. For such short exposure times the contribution of leakage cur- ${ }_{180}$ rents, even when elevated after irradiation, is negligible.

${ }^{4}$ Although all observations support it, this assumption is not trivial, especially for tails more than 5 sigma away from the mean (i.e. very rare events).
}

up to $100 \mathrm{MGy}$ of dose during their expected operation time of 3 years.

In order to investigate the effect of these high levels of radiation an irradiation campaign has been per formed using the DORIS F4 irradiation facility at DESY and prototypes (AGIPD 0.3, AGIPD 0.4) were irradiated to total doses from 1 to $100 \mathrm{MGy}$.

The test chips have been found to tolerate doses up to at least $10 \mathrm{MGy}$. After irradiation the performance of irradiated chips up to this dose is comparable to that of pristine ones, although an increase in noise between $30 \%$ and $40 \%$ was noted [26], which seems to saturate above approximately 1 MGy. Further irradiation to 100 MGy rendered the chip non-functional ${ }^{5}$. After thermal annealing the functionality of the chip is recovered, albeit with a reduced analog performance which is still under investigation. It should be noted that the irradiation of the chip happened in an accelerated fashion compared to operating conditions (accumulating 100 MGy of dose required about 1 week of irradiation at room temperature), so it can be speculated that the thermal annealing during maintenance periods and when the final detector system is not in use might be sufficient to prevent the chip from

\footnotetext{
${ }^{5}$ Tentatively this happens due to charge accumulation in the oxide layer, which causes a substantial shift of the threshold voltages of the devices.
} 


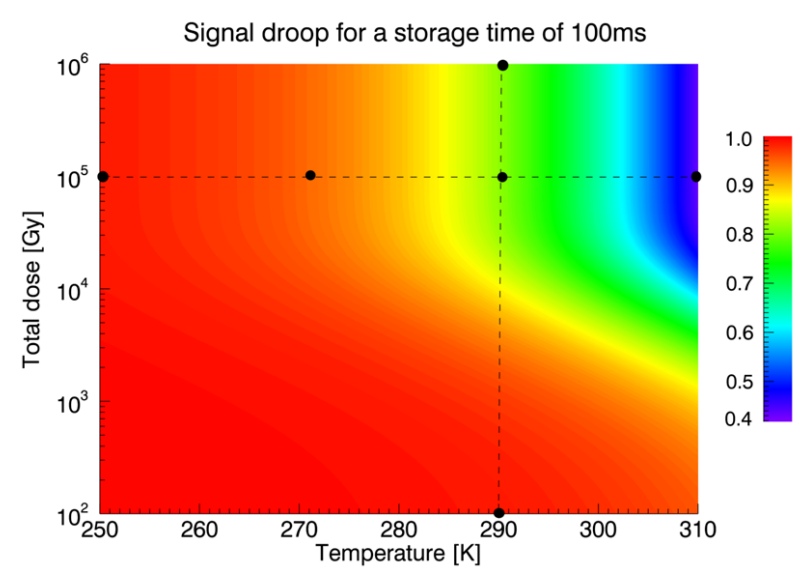

Figure 2: 2D representation of the effect of droop (fraction of charge remaining in the storage cell after 100 ms wait time) $)_{216}$ as function of dose and temperature. The measurements (dark ${ }_{217}$ points) were presented in [27]. Typical storage times for the analog information will be below $12 \mathrm{~ms}$. The data point $\mathrm{at}^{218}$ $100 \mathrm{~Gy}$ corresponds to the situation before irradiation.

losing its functionality.

Additionally it was found that after heavy irradiation and/or at elevated temperatures the signal droop of ${ }^{223}$ the storage cells becomes non negligible. Reducing the ${ }^{224}$ operating temperature has been shown to mitigate this effect [27], and detailed investigations are currently on- ${ }^{226}$ going. In response to these results it was decided to re- ${ }^{227}$ duce the operating temperature to $-20^{\circ} \mathrm{C}$, at which the ${ }^{228}$ droop becomes almost negligible even after irradiation (>99\% of signal remaining) . A 2D overview showing the measured droop at different temperatures and doses for a storage time of $100 \mathrm{~ms}$ is shown in Figure 2. The ${ }^{232}$ regions not covered by measurement points were extrapolated assuming the influence of temperature and dose are independent of each other, described by exponential ${ }^{235}$ functions with a constant offset and their effect is mul- ${ }^{236}$ tiplicative. An elaborate measurement campaign to in- ${ }^{237}$ vestigate the behavior of the droop in detail is currently ${ }^{23}$ ongoing.

\subsection{Interface electronics}

The interface electronics comprises of all the elec- ${ }^{242}$ tronics between the ASICs, which are specially devel-243 oped for AGIPD, and the data acquisition system, which 244 is a common development for all detector systems at the European XFEL.

It can be roughly subdivided into two parts: the control system and the read-out system, both of which are detailed below.

In the mechanical layout, which is detailed later on, care was taken to place as much of the interface electronics as possible outside of the detector vacuum. In this way a forced air stream cooling concept can be realized, which minimizes bulky mechanics outside the detector vacuum and increases the serviceability of electronic components.

\subsubsection{Control system to operate the ASIC and select the best bunches (veto)}

The external control system hardware has been de- $D$ veloped using MTCA.4 crate standards which are common to all European XFEL detectors [28]. An FPGA in the control part of the camera head receives control line signals, performs bookkeeping of free storage cells, and instructs the ASICs which storage cell to use next. The bookkeeping information will be added to the main data stream. In this way it is known to which storage cell a bunch was assigned for later off-line data processing.

An intelligent power supply outside the experimental hutch delivers the power for the ASICs, approximately $500 \mathrm{~A}$ at $1.5 \mathrm{~V}$, and interface electronics and the high voltage of $500 \mathrm{~V}$ or more to the sensors. The control system communication will be based on $10 / 100 \mathrm{Mb}$ Eth ernet using the TCP/IP protocol and will connect both the camera head and the power system. Within the detector head the (slow) control information is distributed and collected by a multi-branch $\mathrm{I}^{2} \mathrm{C}$ network.

There is an additional PCB board in the vacuum connecting the electronics outside of the vacuum to the modules inside. This vacuum board's purpose is the routing of the analog signal lines to the boards described above and the regulation of the voltage on the power lines for the ASICs.

\subsubsection{Analog and digital read-out electronics}

Directly outside the vacuum vessel, a system of ana$\log$ PCBs perform line reception, pickup noise filtering 


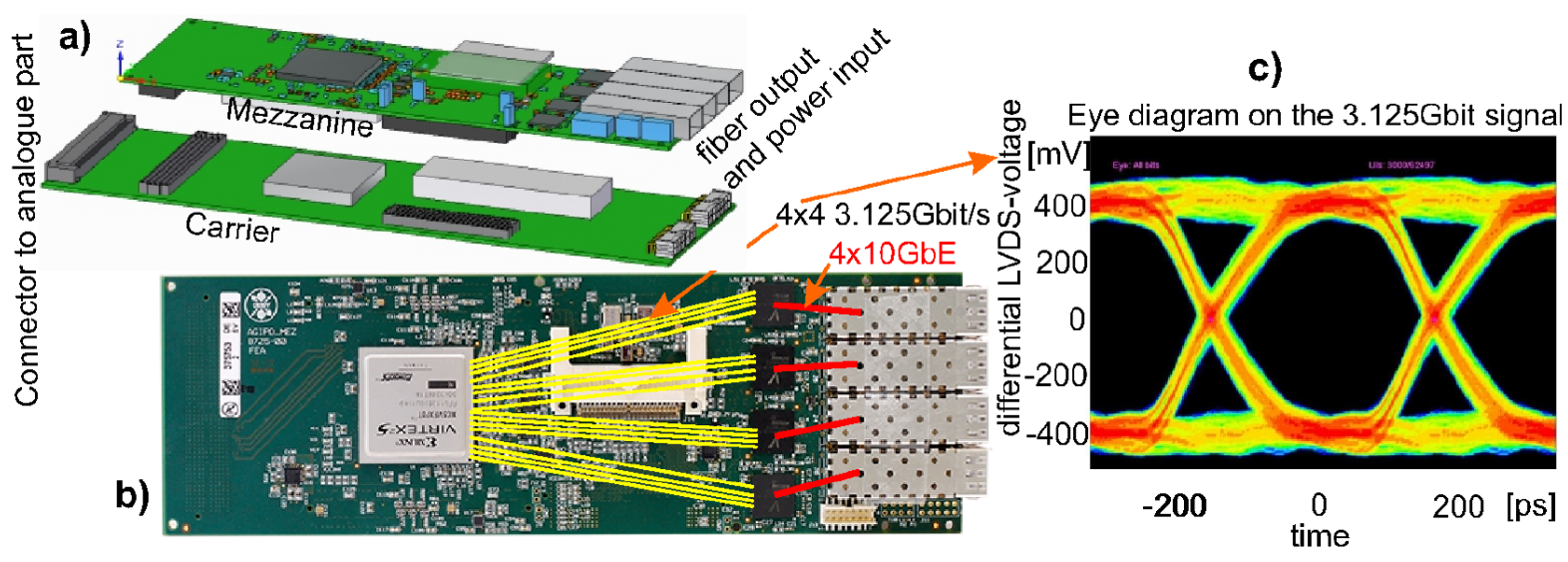

Figure 3: Digital PCB assembly for a module: (a) the mechanical concept, (b) the mezzanine card (c) the eye diagram of the high speed interconnect (3.125 Gbit/s). Image reproduced from [32].

and digitization of the analog signals from the ASICs.270 A single stage filter suppresses the noise at the $33 \mathrm{MHz} 271$ sampling frequency by $3 \mathrm{~dB}$ and settles to better than 272 11 bits accuracy. The analog system also drives the digitized signal to a stack of digital boards which then handle ${ }^{273}$ the communication with the outside world.

The signal of two ASICs is digitized by one AD $9257_{275}^{6}$ fast serial output ADC, which is commonly used in med- 276 ical imaging applications. The data rate per $\mathrm{ADC}$ is ${ }_{277}$ $64 \times 64 / 4 \times 352 \times 14 \times 2 \times 10 \mathrm{bits} / \mathrm{s}^{7} \approx 700 \mathrm{Mbit} / \mathrm{s}$.

The digital PCBs handle the datastream of all $64_{279}$ ADCs of the corresponding module, $45 \mathrm{Gbit} / \mathrm{s}$ in total. ${ }_{280}$ The core of the digital design is a VIRTEX-5 FPGA, ${ }_{281}$ which allows limited data sorting before transmitting $i_{282}$ to the data acquisition (DAQ) system. The digital elec-283 tronic PCB assembly is shown in figure 3.

In addition to the pixel data, the bunch number associated with each storage cell and debug information for ${ }_{286}$ service purposes is transferred to the DAQ system. 287

One of the digital boards is designed as a functional mezzanine board, which is reused for other detector projezess with high speed data readout such as LAMBDA $[29,30]_{289}$ and PERCIVAL [31]. Therefore four $10 \mathrm{GbE}$ outputs $_{290}$ are integrated on the mezzanine, of which AGIPD uses ${ }_{291}$ only one. The 1 Megapixel AGIPD system will deliver

\footnotetext{
${ }^{6}$ Each AD9257 component contains 8 ADC circuits.

${ }^{7}$ Pixels per chip / ADCs per chip x Storage cells x bits per sample $\mathrm{x}(2=$ analog and gain information $) \mathrm{x}$ trains per second.
}

$80 \mathrm{Gbit} / \mathrm{s}$ to the off-detector DAQ system on 16 links in total. Each link is a $10 \mathrm{GbE} / \mathrm{UDP}$ link and will be used with an average data rate of approximately $5 \mathrm{Gbit} / \mathrm{s}$.

\subsection{Data Acquisition (DAQ) system}

The off-detector DAQ is developed as a common component for use with all detectors at European XFEL beamlines. For the 2D cameras and potentially other sys tems (digitizers, fast ADCs, etc.), an ATCA data acqui sition train builder card [33] is being developed to receive, sort and reject data of cameras with 1 Megapixel or more. Data from two AGIPD modules is received by an input train builder FPGA which performs additional module specific data sorting before storing to DDR memory. Final sorting to full 1 Megapixel images and full trains is performed from the input memory to the DDR2 memory of an output FPGA. The latter then sends the bunch ordered images and additional data to a PC-farm for further processing and archiving.

\subsection{AGIPD mechanics}

The basic idea of the mechanical concept is to separate the actual sensing elements (modules), which will be operated in the detector vacuum ${ }^{8}$, from the interface

\footnotetext{
${ }^{8}$ From experience with experiments at the Linear Coherent Light Source (LCLS) it is recommended to separate the vacuum of the sample chamber from the detector vacuum in order to avoid the accumulation of sample residue on sensitive parts of the detector.
} 


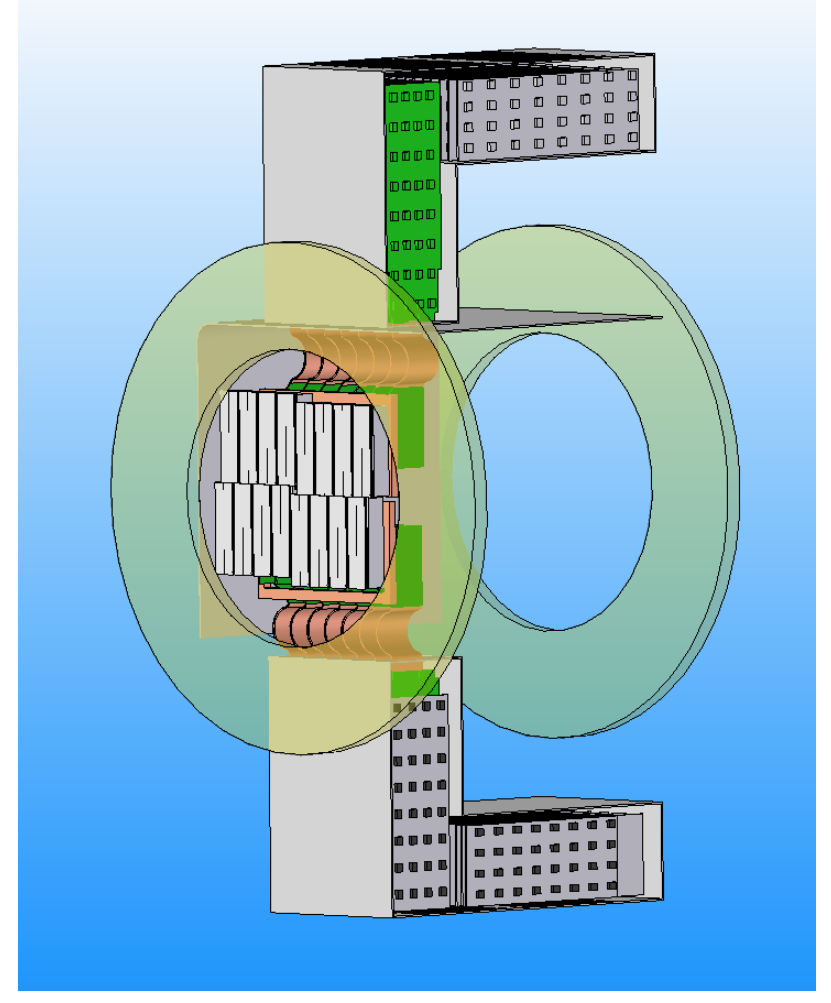

Figure 4: Concept of the upstream detector of 1 Megapixel $^{317}$ (16 modules). electronics, which will be outside of the vacuum.

In order to reduce the amount of space occupied 322 along the beam axis, the electronics was designed in an 323 angled shape, and form so called wings. Each wing will 324 feature a closed loop air stream inside it to transport the 325 heat dissipated in the interface electronics (about $500 \mathrm{~W}$ ) ${ }^{326}$ to a special, water cooled heat exchanger in order to min-327 imize the heat dissipated to the air of the experimental 328 area.

The detector will consist of one main (upstream) de- ${ }^{330}$ tector of 1 Megapixel, shown in Figure 4, which consists ${ }^{331}$ of 4 quadrants. In order to increase the angular coverage towards the central beam, the SPB beamline at the Euro- ${ }^{332}$ pean XFEL will additionally employ a $128 \mathrm{k}$ downstream $_{333}$ detector consisting of 2 modules, as shown in Figure 5. ${ }_{334}$

To achieve the best possible scientific outcome the 335 four quadrants of the main detector and the two modules 336 of the downstream detector need to be movable, primar-337 ily to adjust the size of the central hole.

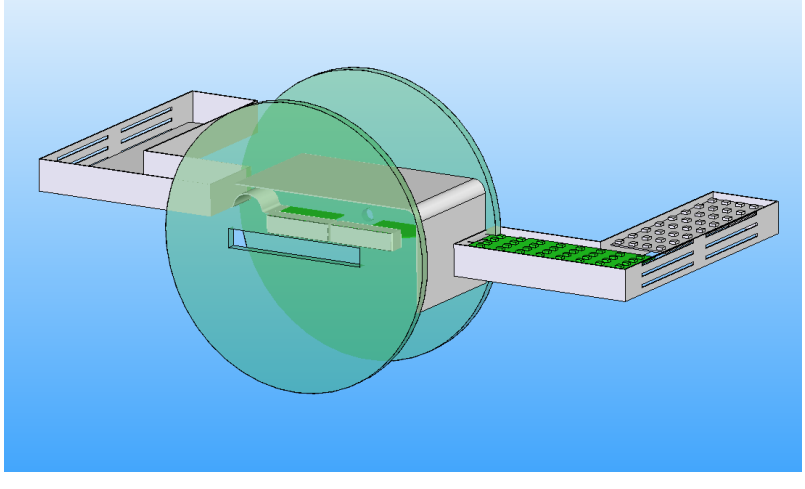

Figure 5: Concept of the downstream detector of $128 \mathrm{k}$ pixels ( 2 modules).

\subsubsection{Modules}

The basic building block of the detector system is the so called module, where each module is, in principle, an independent detector unit.

A so called hybrid is formed by an array of $2 \times 8$ ASICs which are bump bonded to the monolithic pixelated silicon sensor described above. The bump bonding will be performed at PSI using their well developed inhouse technology [34].

The hybrid is then glued onto a so-called sensor board made from low temperature co-fired ceramics (LTCC) material. The hybrid is then electrically connected to this LTCC board by wirebonding to form the bare module. Optionally a heatspreader of up to $500 \mu \mathrm{m}$ thickness can be mounted in between the hybrid and the sensor board to improve temperature homogeneity and to take up stresses from the mismatching thermal expansion coefficients of silicon and the LTCC material.

To complete the full module the bare module needs to be mounted onto the quadrant cooling block and the vacuum board needs to be connected.

\subsubsection{Cooling system}

As the total power dissipation inside the vacuum will be around $1 \mathrm{~kW}$, a cooling concept using a liquid coolant was adopted. The main coolant will be provided by a cooling plant outside the experimental hutch and has to be brought to, and extracted from, the experimental area via a dedicated pipe system. 


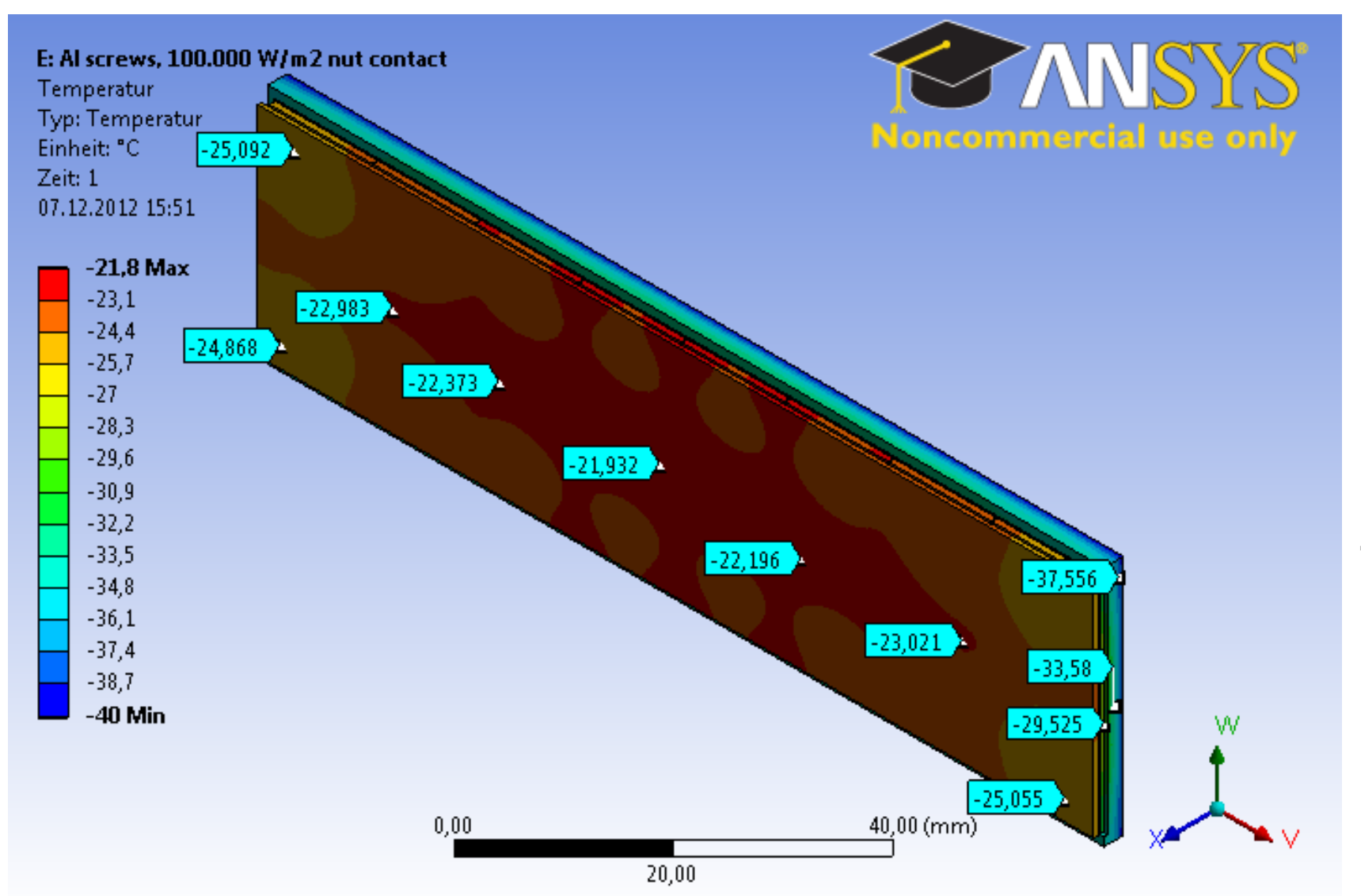

Figure 6: Thermal simulation of a bare module connected to the cooling block. The cooling block is at $-40^{\circ} \mathrm{C}$, the warmest point at $-21.9^{\circ} \mathrm{C}$. The temperature spread within the sensor is $3.5^{\circ} \mathrm{C}$.

First the main coolant will flow into the four quad-356 rant cooling blocks in parallel. The cooling blocks have 357 internal cooling channels that meander through the avail-358 able volume before leaving the quadrant cooling blocks.359 The outflowing coolant will then be fed into a secondary ${ }_{360}$ cooling block that, in turn, removes the heat from the 361 voltage regulators on the vacuum board.

This concept is beneficial for the temperature uniformity of the ASICs, as it isolates the ASICs from the 363 heat load of the vacuum boards. Additionally the voltage 364 regulators only need to be stabilized in temperature; the ${ }^{365}$ temperature uniformity is of secondary importance. $\quad 366$

As stated above, it is intended to operate irradiated ${ }^{367}$ ASICs at a temperature of $-20^{\circ} \mathrm{C}$ or below to reduce the $\mathrm{e}^{368}$ droop on the analog storage cells. Simulations of the sensor stack (Figure 6) show that the temperature difference between the hottest and the coldest point on the sensor is less than $4^{\circ} \mathrm{C}$, and that all ASICs operate at a temperature of $-22^{\circ} \mathrm{C}$ or lower when the main coolant is at a temperature of $-40^{\circ} \mathrm{C}$. Slight variations of these values are expected, as the coolant temperature will increase while passing through the quadrant. The largest temperature drop, $>10^{\circ} \mathrm{C}$, happens in the $2.4 \mathrm{~mm}$ thick LTCC material of the sensor board.

In order to maximize the temperature stability of the cooling system, a chiller system using a bath design with a volume of more than 201 was chosen. A polydimethyl siloxane $\left(\mathrm{PDMS}^{9}\right.$ ) based coolant, which fulfills the necessary requirements of low viscosity and chemical inertness in the anticipated temperature range, will be used.

\footnotetext{
${ }^{9} \mathrm{PDMS}$ is commonly known as silicone oil
} 


\section{Summary and outlook}

The AGIPD project, a joint detector development 411 program by DESY, PSI and the universities of Hamburg ${ }_{412}$ and Bonn, is progressing and the first module is expected ${ }_{413}$ to be operational before the end of 2013 .

The test chips allowed the investigation of the performance of individual ASIC components, including their ${ }_{416}$ radiation hardness, and the results have proven invalu- ${ }_{417}$ able for the design of the full scale chip AGIPD 1.0, the 418 submission of which is immanent.

The full 1 Megapixel system will be deployed at the ${ }_{420}$ European XFEL in 2015 for commissioning and calibra- 421 tion and will be available for user operation at "Day 1 "422 in 2016.

\section{References}

[1] M. Altarelli et al., European X-ray Free Electron Laser. ${ }^{427}$ Technical Design Report, ISBN 978-3-935702-17-1 (2006).

[2] T. Tschentscher et al., Layout of the X-Ray Systems at 430 the European XFEL, TECHNICAL NOTE XFEL.EU 431 TN-2011-001 (2011) DOI: 10.3204/XFEL.EU/TR-2011-001.

[3] B. Henrich et al., The adaptive gain integrating pixel detector AGIPD a detector for the European XFEL, Nucl. Instr. and Meth. A, DOI: 10.1016/j.nima.2010.06.107.

[4] X. Shi et al., Challenges in chip design for the AGIPD ${ }_{438}$ detector, Nucl. Instr. and Meth. A 624(2) 2010 387-391, DOI: $10.1016 /$ j.nima.2010.05.038.

[5] G. Potdevin et al., Performance simulation of a detector ${ }^{44}$ for 4th generation photon sources: The AGIPD, Nucl. ${ }_{442}$ Instr. and Meth. A 607(1) 2009 51-54, DOI: 10.1016/j.nima.2009.03.121.

[6] R. Neutze et al., Potential for biomolecular imaging with femtosecond X-ray pulses, Nature 406, 752-757 2000, doi:10.1038/35021099.

[7] H. Graafsma, Requirements for and development of 2 dimensional X-ray detectors for the European X-ray Free Electron Laser in Hamburg, J. Inst. 42009 P12011, doi:10.1088/1748-0221/4/12/P12011.
[8] A. Mancuso et al., Conceptual Design Report: Scientific Instrument SPB, 2011, TR-2011-007 DOI: 10.3204/XFEL.EU/TR-2011-007.

[9] A. Madsen et al., Conceptual Design Report: Scientific Instrument MID, 2011, TR-2011-008 DOI: 10.3204/XFEL.EU/TR-2011-008.

[10] J. Becker and H. Graafsma, Advantages of a logarithmic sampling scheme for XPCS experiments at the European XFEL using the AGIPD detector, J. Inst. 72012 P04012, DOI:10.1088/1748-0221/7/04/P04012.

[11] J. Schwandt et al., Optimization of the Radiation Hardness of Silicon Pixel Sensors for High X-ray Doses using TCAD Simulations, J. Inst. 72012 C01006 DOI:10.1088/1748-0221/7/01/C01006.

[12] J. Schwandt et al., Design of the AGIPD Sensor for the European XFEL, to be published in Proceedings of the 14th International Conference on Radiation Imaging Detectors 1 - 5 July 2012, Figueira da Foz, Portugal, and arXiv:1210.0430.

[13] J. Zhang et al., Study of radiation damage induced by $12 \mathrm{keV}$ X-rays in MOS structures built on high-resistivity n-type silicon, J. Synchrotron Rad. (2012) 19 340-346, doi:10.1107/S0909049512002348

[14] J. Zhang et al., Study of X-ray radiation damage in silicon sensors, J. Inst. 62011 C11013, doi:10.1088/1748-0221/6/11/C11013.

[15] J. Zhang et al., Investigation of X-ray induced radiation damage at the $\mathrm{Si}-\mathrm{SiO} 2$ interface of silicon sensors for the European XFEL, http://arxiv.org/abs/1210.0427.

[16] J. Becker, D. Eckstein, R. Klanner, G. Steinbruck, Impact of plasma effects on the performance of silicon sensors at an X-ray FEL, Nucl. Instr. and Meth. A 615(2) 2010 230-236, doi:10.1016/j.nima.2010.01.082.

[17] J. Becker, Signal development in silicon sensors used for radiation detection, $\mathrm{PhD}$ thesis, Hamburg University, July 2010, DESY-THESIS-2010-033.

[18] J. Becker, K. Gärtner, R. Klanner, R. Richter, Simulation and experimental study of plasma effects in planar silicon sensors, Nucl. Instr. and Meth. A 624(3) 2010 716-727, doi:10.1016/j.nima.2010.10.010.

[19] G. Potdevin, U. Trunk, H. Graafsma, HORUS, an HPAD X-ray detector simulation program, J. Inst. 4 2009 P09010, doi:10.1088/1748-0221/4/09/P09010. 
[20] G. Potdevin, H. Graafsma, Analysis of the expected AGIPD detector performance parameters for the European X-ray free electron laser, Nucl. Instr. and Meth. A, doi:10.1016/j.nima.2011.09.012.

[21] J. Becker, D. Pennicard and H. Graafsma, The detector 499 simulation toolkit HORUS, J. Inst. 72012 C10009, doi:10.1088/1748-0221/7/10/C10009.

[22] U. Trunk et al., AGIPD - The Adaptive Gain Integrating Pixel Detector for the European XFEL. Development 503 and Status, Proceedings of Nuclear Science Symposium ${ }^{504}$ and Medical Imaging Conference (NSS/MIC), 23-29 505 Oct. 2011, doi:10.1109/NSSMIC.2011.6154392.

[23] D. Greiffenberg, The AGIPD detector for the European ${ }^{507}$ XFEL, J. Inst. 72012 C01103 doi:10.1088/1748-0221/7/01/C01103.

[24] J. Becker et al., The single photon sensitivity of the Adaptive Gain Integrating Pixel Detector, Nucl. Instr. and Meth. A 694(1) 2012 82-90, doi:10.1016/j.nima.2012.08.008.

[25] D. Greiffenberg et al., Investigation of the noise performance of the AGIPD prototype chips, submitted to J. Inst.

[26] A. Marras, et al., Front end electronics for European XFEL sensor: the AGIPD project,Proceedings of 6th international Workshop on Semiconductor Pixel Detectors for Particles and Imaging (PIXEL2012), September 3 - 7, 2012 in Inawashiro, Japan, to appear in Nucl. Instr. and Meth. A.

[27] U. Trunk, AGIPD - the Adaptive Gain Integrating Pixel Detector for the European XFEL, 2011, IEEE-NSS conference Record, N39-5, ISBN 978-1-4573-0119-0 1950pp.

[28] S. Cook et al., Further Development of the MTCA.4 Clock and Control System for the EuXFEL Megapixel Detectors, TWEPP-2012, Oxford, Great Britain, proceeding to appear in $\mathrm{J}$. Inst.

[29] D. Pennicard et al., Development of LAMBDA: Large Area Medipix-Based Detector Array, J. Inst. 6 C11009, doi:10.1088/1748-0221/6/11/C11009.

[30] D. Pennicard et al., LAMBDA - Large Area Medipix3-Based Detector Array, IWORID 2012, Figueira da Foz, Coimbra, PORTUGAL, accepted for the proceedings to appear in J. Inst.
[31] C. Wunderer, Soft X-Ray Detector Developments at DESY, talk presented on the 11th International Conference on Synchrotron Radiation Instrumentation, 9-13 June 2012, Lyon, France.

[32] M. Zimmer, I. Sheviakov, A versatile High Speed Data Acquisition Module with four 10G-Ethernet Links, IEEE real-time conference, June 11th-15th 2012, Berkeley, USA.

[33] J. Coughlan et al., The data acquisition card for the Large Pixel Detector at the European-XFEL, J. Inst. 6 2011 C12057, doi:10.1088/1748-0221/6/12/C12057.

[34] T. Rohe, et al., Nucl. Instr. and Meth. A 565 (2006) 303, doi:10.1016/j.nima.2006.05.011. 